\title{
Psychometric validation of the work productivity and activity impairment questionnaire in ulcerative colitis: results from a systematic literature review
}

\author{
Aaron Yarlas ${ }^{1 *} \mathbb{D}$, Stephen M. Maher ${ }^{1}$, Martha S. Bayliss ${ }^{1}$, Andrew Lovley ${ }^{1}$, Joseph C. Cappelleri ${ }^{2}$ and
} Marco D. DiBonaventura ${ }^{3}$

\begin{abstract}
Patients with ulcerative colitis, a type of inflammatory bowel disease, report negative impacts of disease symptoms on work-related outcomes, including absenteeism and presenteeism. As a way to better understand the impact of this disease and its treatment on work-related outcomes, the current review examines the use of the Work Productivity and Activity Impairment Questionnaire (WPAI), a patient-reported outcomes measure of absenteeism, presenteeism, and impairment in other activities, in studies of patients with ulcerative colitis. This review assesses the measurement properties of the WPAI in this patient population: its reliability, construct validity, ability to detect change, and responsiveness to effective treatments. Relevant data were extracted from 13 sources (journal articles and conference posters) identified following a systematic review of the published and gray literature. The evidence supports the WPAI as having test-retest reliability (reproducibility) over time; convergent validity, as indicated by moderate correlations with measures of quality of life and moderate-to-strong correlations with measures of disease activity; known-groups validity, as indicated by differences in WPAI scores between patients with active and inactive disease; ability (sensitivity) to detect change, as indicated by substantial improvement in scores for patients who achieve remission, accompanied by substantial worsening of scores for patients who relapse; and, responsiveness to treatment, with improvements in scores following treatments that reduce disease activity. Limitations included a lack of available evidence from randomized-controlled trials that could speak more directly to the WPAl's responsiveness to treatment. In conclusion, we recommend the use of the WPAl for measuring work outcomes in both observational studies and interventional trials that include patients with ulcerative colitis.
\end{abstract}

Keywords: Ulcerative colitis, Inflammatory bowel disease, Work productivity and activity impairment questionnaire, Absenteeism, Presenteeism, Work productivity, Literature review

\section{Introduction}

Ulcerative colitis (UC) is an inflammatory disease of the colon that is characterized by intermittent periods of disease flaring and remission and affects 900,000 patients in the United States alone [1, 2]. Patients with UC experience recurring clinical signs and symptoms, including rectal bleeding, abdominal pain, frequent diarrhea, and an urgent need to defecate. These symptoms are typically assessed in clinical trials using a

\footnotetext{
* Correspondence: ayarlas@qualitymetric.com

'Optum, 1301 Atwood Avenue, Suite 311N, Johnston, RI 02919, USA

Full list of author information is available at the end of the article
}

disease activity index, such as the Mayo score [3] or the Simple Clinical Colitis Activity Index (SCCAI), [4] among others.

What these disease activity measures often fail to capture, however, is the impact of these clinical signs and symptoms on the everyday functioning of patients with UC. One aspect of functioning likely to be affected by recurrent symptoms is work-related outcomes (WRO), such as absenteeism and impairment in productivity at work (i.e., presenteeism). Several studies have used individual or group interviews of patients with inflammatory bowel disease (IBD; inclusive of both UC 
and Crohn's disease) to elicit patients' input on the effect of the disease on patients' everyday lives, including work experiences [5-10]. Patients in these studies discussed missing work because of disease-related pain, fatigue, or other symptoms [5, 6, 8-10].

Further, in all of these studies, patients described the negative impact on their work performance due to these symptoms, including the difficulty of accessing a toilet and avoiding meetings or interactions with colleagues to hide their symptoms and frequency of toilet use. The increase of absenteeism and presenteeism has been shown to limit employment opportunities for patients with UC; several studies have reported rates of unemployment and use of disability benefits for patients with IBD that were typically two-to-three times higher than those for matched general population controls [11-14]. Further examination of unemployment and disability rates found that these differences disappear when only including IBD patients who are asymptomatic or in remission $[15,16]$.

Collectively, these results indicate that patients with active UC have impaired WRO and that inducing remission may improve these outcomes. Thus, it is important that clinical trials capture the degree to which the studied treatment may be able to improve WRO. Since objective data on WRO (e.g., absentee information from employment databases) are difficult to obtain in a clinical trial setting, self-reported measures are typically used to assess the impact of disease and treatment on absenteeism and presenteeism. Several patient-reported outcomes measures (PROMs) have been developed for this purpose; among the most frequently used is the Work Productivity and Activity Impairment questionnaire (WPAI) [17].

The WPAI measures the impact of health problems on absenteeism, presenteeism, overall work performance, and non-work activities. The WPAI has been shown to be reliable, valid, and responsive when used with patients across several disease areas, including those with gastrointestinal conditions (e.g., irritable bowel syndrome, $[18,19]$ gastroesophageal reflux disease [20], and Crohn's disease [21, 22]). However, there has not (to our knowledge) yet been an examination of the measurement properties of the WPAI when used with samples of patients with UC; as such, we believe that this article is the first to do so.

The objective of this paper is to report results from the first systematic literature review on the measurement properties of the WPAI when used with UC patients. Studies included in this review were identified within both the published and unpublished (or "gray") literature. Evidence was synthesized across identified studies for the purpose of examining evidence for the instrument's reliability, construct validity, ability to detect change, and responsiveness to treatment for the UC patient population.

\section{Methods \\ WPAI}

The WPAI (presented in Additional file 1) is a self-administered six-item survey designed to measure the impact of a person's health problems on WRO over the previous seven days [17]. This includes work time missed (absenteeism), impaired productivity at work (presenteeism), overall work impairment (OWI; combined absenteeism and presenteeism), and impairment in non-work-related activities due to health problems (activity impairment), over the previous seven days.

Depending on how questions are framed, the WPAI can measure the impact of general health problems (WPAI-GH), or the impact of a specific health problem. In the latter case, the name of the condition is usually included; for example, if subjects are asked to answer the questions regarding the impact of UC specifically (rather than "health problems" generically, as in the WPAI-GH), the instrument would be referred to as the WPAI-UC.

\section{Systematic literature review \\ Search sources and terms}

The literature search and selection process adhered to guidelines described in the Preferred Reporting Items for Systematic Reviews and Meta-Analyses (PRISMA) Statement [23]. In particular, we conducted searches of PubMed, Embase, and the Cochrane Register of Controlled Trials (CENTRAL) for publications in peer-reviewed journals. We also searched for posters and presentations at peer-reviewed national and international conferences on topics of gastrointestinal diseases and measurement of patient-reported outcomes (PRO) using Embase and the International Society for Pharmacoeconomics and Outcomes Research (ISPOR) scientific presentations database. All searches were performed in November 2017. Search terms were designed to capture studies in which the WPAI was administered to patients with UC or IBD more generally (see Additional file 1 for full search strings).

In addition, we used the search engine on the ClinicalTrials.gov website, using the keywords "work productivity" and "WPAI" with conditions of "inflammatory bowel disease" and "ulcerative colitis." We also reviewed references listed on the WPAI developer's webpage (http://www.reillyassociates.net/WPAI_References.html) and those cited in selected records. The protocol outlining the search strategy is available upon request from the authors. 


\section{Selection of records (articles and posters)}

Screening of each record at each stage of review was conducted by at least two of three independent reviewers (AY, SM, and AL). Discrepancies at any stage of review were discussed by all three reviewers until consensus was reached.

Initial screening was based on articles' titles and abstracts. Full-text articles/posters of records not excluded during title/abstract screening were retrieved to perform a further review. Records for which the full-text was available online or for purchase were retrieved directly. For items not directly available via these means, authors were contacted via email (and phone, when possible) in an attempt to retrieve the full text.

At each screening phase, records were selected if they met (or, during abstract screening, did not clearly fail to meet) the following inclusion criteria: published in English, and provided quantitative WPAI-GH or WPAI-UC data for adult patients with UC (or within a sample of IBD patients, with data reported separately for a UC patient subgroup) that could be used to assess the instrument's reliability, validity, responsiveness, or sensitivity to treatment. Data reported numerically were extracted directly from selected records and added to a database to be summarized. Data reported only graphically (i.e., in a figure) were extracted using WebPlotDigitizer-Desktop, version 2.8 (https://automeris.io/WebPlotDigitizer), a computer program that uses the spatial distances of the axes to determine the numeric values provided.

\section{Assessment of measurement properties of the WPAI in UC}

\section{Reliability}

Given that each of the single WPAI items captures a distinct and independent construct, internal consistency was not considered for review as it requires multiple items per construct. Test-retest reliability, or reproducibility, of WPAI domains was evaluated from studies assessing the magnitude of change in WPAI domain mean scores across two time points for patients with stable disease activity during the interval (e.g., were not in remission at either time, or were in remission at both times). Evidence that changes in WPAI domains were small, and do not exceed established clinically important change (CIC) thresholds, would support the instrument as having adequate test-retest reliability.

\section{Construct validity}

Construct validity was assessed through both convergent validity and known-groups validity. Convergent validity assesses whether instruments that purport to measure the same construct (or conceptually similar constructs) show strong concordance. Convergent validity was examined by evaluating correlations between WPAI domain scores and scores from instruments measuring conceptually related constructs, including health-related quality of life (HRQoL) and UC disease activity. Evidence for acceptable convergent validity were correlation coefficient values $\geq|0.40|$, with correlations $<|0.40|$ but $\geq|0.30|$ not considered as evidence to dismiss convergent validity, as recommended for determination of this property when using PRO measures [24]. Known-groups validity assesses whether the scores from the instrument differ across groups known to differ on that construct (or conceptually similar construct). Known-groups validity was assessed by examining the magnitude of differences in WPAI domain scores between groups that were known to differ in UC disease activity (e.g., active disease vs. remission), or specific health-relevant symptoms (e.g., fatigue).

\section{Ability to detect change}

The ability of the WPAI domains to detect changes in UC disease activity was evaluated by assessing the magnitude of change in WPAI domain mean scores for patients showing clinically meaningful changes in activity status (i.e., a change from active disease to remission, or vice-versa, based on pre-specified criteria from a disease activity index). Evidence that changes in WPAI domains exceed a level that indicates clinical importance would support the instrument as able to detect change. CICs have been conceptually defined as the smallest change in score which patients perceive as beneficial and for which a clinician would recommend a change in the patient's care [25]. Evaluation of within-patient change in scores for the WPAI-CD (i.e., the WPAI where "health problems" is replaced with "Crohn's disease") using both distribution-based methods and anchor-based methods (defined by changes on the $\mathrm{CD}$ activity index [CDAI] [26]) found that a change of $7 \%$ in each WPAI-CD domain corresponds to a clinically meaningful change in CD patients' disease activity $[21,27]$. Despite the lack of direct evaluation of a CIC for the WPAI-UC, given the similarities between symptoms of the two conditions, previous research on the WPAI in UC patients have adopted these CIC thresholds [28, 29].

\section{Responsiveness to treatment}

The responsiveness of the WPAI domains to treatments shown to be effective for reducing UC activity was evaluated by assessing the magnitude of change in WPAI domain mean scores from baseline to post-treatment assessment. Evidence that changes in WPAI domains exceed established CIC thresholds would support the instrument as responsive to treatment. 


\section{Results}

\section{Literature review}

The number of records retrieved from each queried source, the number excluded from the review at each stage of selection, and the number selected for review, for both published articles and unpublished conference posters, are reported in the PRISMA flow chart in Fig. 1. Data from 13 records - eight articles [29-36] and five posters [37-41] - that met all selection criteria were identified from the literature search.

We used two tools to assess the quality of these 13 studies. The first tool, the Joanna Briggs Institute (JBI) critical appraisal checklist for analytical cross sectional studies, [42] was used to evaluate the quality of the eight non-interventional cross-sectional studies [30-35, 38, 39] identified from our literature search. The second tool, the National Institute of Health (NIH) quality assessment tool for observational cohort and cross-sectional studies, [43] was used to evaluate the quality of the five open-label non-comparative interventional studies [29, 36, 37, 40, 41] identified in our literature search. Neither of these tools provides a clear-cut algorithm for determining the quality of the study or for deciding whether the study should be included in or excluded from a review; rather, the decision is left to the reviewer based on the pattern of checklist responses.
Based on the patterns of responses to the JBI checklist, each of the eight cross-sectional studies was appraised as having sufficient quality to be rated as "include", and so data from all of these studies were included in the review. Based on the patterns of responses to the checklist within the NIH quality assessment tool, each of the five open-label non-comparative interventional studies was given a quality rating of either "good" or "fair", and so data from all of these studies were included in the review. Sample and design characteristics of the 13 reviewed studies are presented in Table 1.

At least one of the, and often several, authors of the 24 unavailable posters were contacted to obtain the posters. From these contacts, replies were received from three authors who declined to share results: one claimed their data were proprietary content, one stated that the data were already published (these published data have been included in this review [29]), and one stated that the data were qualitative in nature. For the remaining posters, no authors responded to our requests after sending three separate emails.

\section{Reliability}

Test-retest reliability of WPAI domains was evaluated based on results from one study [37] that compared scores at the start and end of an open-label maintenance

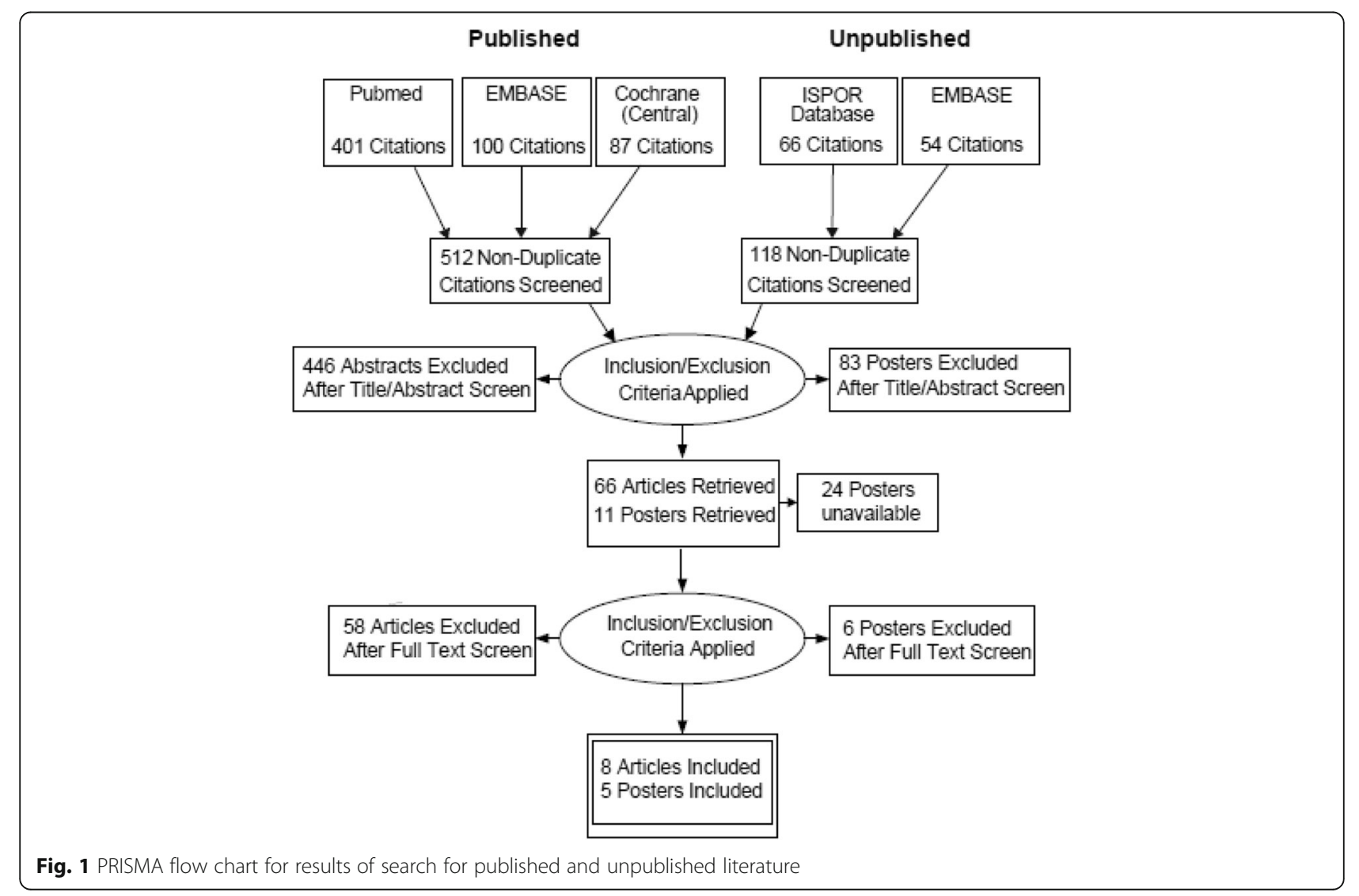




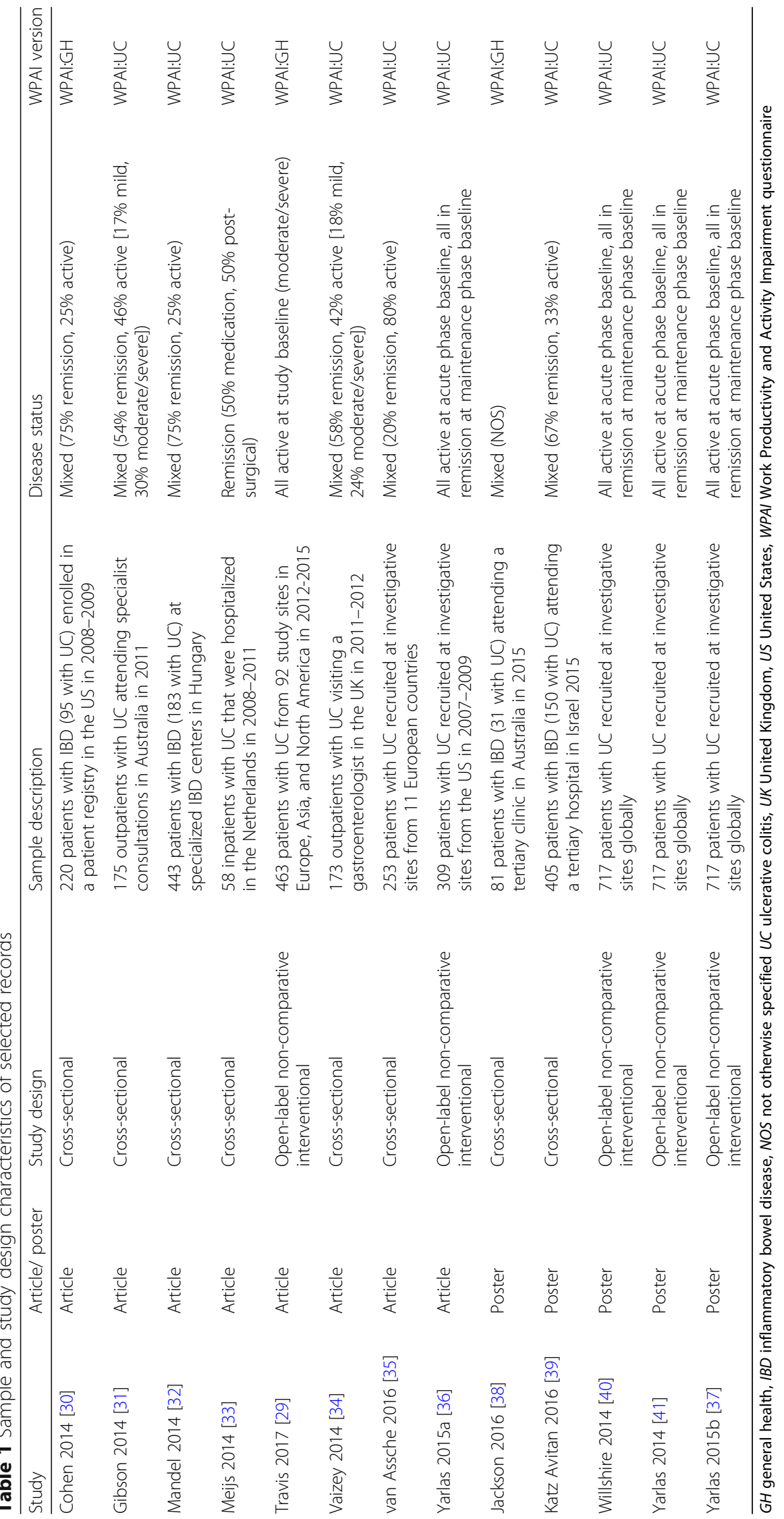


Table 2 Changes in WPAI scores over time for patients with no change in UC disease activity status

\begin{tabular}{|c|c|c|c|c|c|c|c|}
\hline \multirow[b]{2}{*}{ Study } & \multirow[b]{2}{*}{$\mathrm{N}$} & \multirow[b]{2}{*}{ Status at both assessments } & \multirow[b]{2}{*}{ Interval } & \multicolumn{4}{|c|}{ Change in mean WPAI scores from Time 1 to Time 2} \\
\hline & & & & Absenteeism & Presenteeism & OWl & Activity Impairment \\
\hline Yarlas 2015b [37] & 45 & Complete remission $^{\mathrm{a}}$ & 12 months $^{c}$ & -0.5 & -4.2 & -4.8 & 0.2 \\
\hline Yarlas 2015b [37] & 53 & Partial remission ${ }^{b}$ & 12 months $^{c}$ & -4.3 & -1.1 & -4.9 & -1.6 \\
\hline \multicolumn{4}{|c|}{ Weighted Mean differences $^{d}$} & -2.6 & -2.5 & -4.9 & -0.8 \\
\hline
\end{tabular}

OWI Overall Work Impairment, UC ulcerative colitis, WPAI Work Productivity and Activity Impairment questionnaire

${ }^{a}$ Complete remission defined as UC-DAl total score $\leq 1$, rectal bleeding and stool frequency components $=0$, and $\geq 1$-point reduction in endoscopy score from induction phase baseline

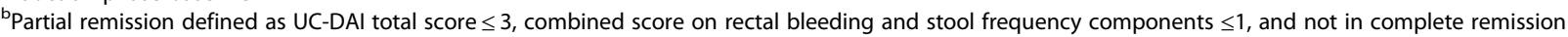

${ }^{C}$ Receiving multimatrix mesalamine $2.4 \mathrm{~g} /$ day once daily

${ }^{\mathrm{d}}$ Weighted by sample size

treatment phase period for patients whose remission status (partial remission or complete remission) was unchanged, as determined by scores on the Ulcerative Colitis - Disease Activity Index (UC-DAI) [44]. As reported in Table 2, differences in percentages, even across 12 months, were less than $5 \%$ for each domain, none of which exceeded the CIC threshold of $7 \%$ for clinically meaningful change. No measure of association (e.g., intraclass correlation coefficient) was reported for these data.

\section{Convergent validity}

\section{Correlations with criterion measures of HRQoL}

Evidence for convergence between WPAI domains and measures of HRQoL was evaluated based on results from one study of patients with UC [36]. This study reported Spearman rank-order correlation coefficients between WPAI domains and subscales from two HRQoL measures: the Short Inflammatory Bowel Disease Questionnaire (SIBDQ), [45] and the SF- $12^{\circ}$ Health Survey, version 2 (SF-12v2) [46]. Both of these measures were deemed as appropriate for testing the convergent validity of the WPAI because their parent instruments (the SF-36 and the IBDQ, respectively) had been included as criteria for testing the convergent validity of the WPAI-CD [21]. Because higher subscale scores for these two measures indicate better HRQoL, and higher scores on WPAI indicate compromised work productivity or activity impairment, it was expected that all correlation coefficients would be in the negative direction.

As reported in Table 3, all correlations between the domains of the WPAI and the SIBDQ were negative in

Table 3 Convergent validity: Spearman rank-order correlation coefficients between WPAI domains and criterion measures of healthrelated quality of life

\begin{tabular}{|c|c|c|c|c|}
\hline \multirow[b]{2}{*}{ Criterion Measure } & \multicolumn{4}{|l|}{ WPAI Domain } \\
\hline & Absenteeism & Presenteeism & Overall Work Impairment & Activity Impairment \\
\hline \multicolumn{5}{|l|}{$\overline{S I B D Q}$} \\
\hline Bowel symptoms & -0.47 & -0.68 & -0.68 & -0.55 \\
\hline Systemic symptoms & -0.13 & -0.31 & -0.31 & -0.46 \\
\hline Emotional function & -0.15 & -0.40 & -0.38 & -0.49 \\
\hline Social function & -0.36 & -0.64 & -0.63 & -0.68 \\
\hline Median correlation & -0.26 & -0.52 & -0.51 & -0.52 \\
\hline \multicolumn{5}{|l|}{ SF-12v2 } \\
\hline Physical functioning & -0.22 & -0.28 & -0.28 & -0.35 \\
\hline Role physical & -0.38 & -0.57 & -0.57 & -0.50 \\
\hline Bodily pain & -0.52 & -0.54 & -0.54 & -0.55 \\
\hline General health & -0.07 & -0.15 & -0.12 & -0.34 \\
\hline Vitality & -0.33 & -0.31 & -0.31 & -0.44 \\
\hline Social functioning & -0.37 & -0.35 & -0.36 & -0.44 \\
\hline Role emotional & -0.35 & -0.46 & -0.46 & -0.41 \\
\hline Mental health & -0.18 & -0.17 & -0.17 & -0.33 \\
\hline Median correlation & -0.34 & -0.33 & -0.34 & -0.43 \\
\hline
\end{tabular}

SF-12v2 SF-12v2 Health Survey, SIBDQ Short Inflammatory Bowel Disease Questionnaire, WPAI Work Productivity and Activity Impairment questionnaire Note: all data in this table are from Yarlas et al (2015a) [36]

Values printed in BOLD indicate correlations satisfying the definition of convergent validity $(\geq|0.40|)[24]$ 
direction, as expected, ranging from -0.13 to -0.68 (overall median $=-0.47$ ). All WPAI domains met criteria for convergence $(\geq 0.40)$ [24] with the SIBDQ Bowel symptoms subscale. All WPAI domains, with the exception of absenteeism, were convergent with the SIBDQ Social function subscale. Presenteeism and activity impairment domains were convergent with the SIBDQ Emotional function subscale, while only the activity impairment domain was convergent with the SIBDQ Systemic symptoms subscale. Across SIBDQ subscales, convergence was supported for presenteeism, OWI, and activity impairment (median correlations ranging from -0.51 to -0.52 ).

All inter-scale correlations between WPAI domains and SF-12v2 subscale scores were in a negative direction and ranged from -0.07 to -0.57 (overall median $=-$ 0.35). The WPAI domains showed the greatest degree of convergence with the Bodily pain subscale (all domains meeting criteria), followed by the Role physical and Role emotional subscales (presenteeism, OWI, and activity impairment domains met criteria for both). These associations with SF-12v2 Role limitations subscales would be expected, as they assess similar constructs as the WPAI domains, namely the impact of health problems on patients' work productivity and ability to engage in other activities [46]. Across SF-12v2 subscale scores, convergence was supported only for the activity impairment domain (median correlation of -0.43), though median correlations for the other three domains (ranging from -0.33 to -0.34 ) were large enough that convergent validity cannot be dismissed.

\section{Correlations with criterion measures of disease activity}

Evidence for convergence between measures of disease activity and the domains of the WPAI was evaluated based on three studies [32, 38, 41] of UC patients that reported Spearman rank-order correlation coefficients between WPAI domains and scores from one of three measure of disease activity: the partial Mayo score, [3] the SCCAI, [4] and the UC-DAI [44]. Because higher scores for each of these measures indicate increased disease activity, it was expected that all correlation coefficients with WPAI would be positive values.

As reported in Table 4, all correlations were positive. Inter-scale correlations between WPAI domains and disease activity measures ranged from 0.32 to 0.85 (median $=0.45$ ). The OWI domain was convergent with disease activity measures in all three studies, while the presenteeism and activity impairment domains were convergent with disease activity measures in two of the three studies (correlations with the absenteeism domain were only reported in two studies; convergence with disease activity was found in one of these). Across studies, convergence with disease activity was supported for presenteeism, OWI, and activity impairment (median correlations ranging from 0.43 to 0.60 ), with the median correlation for absenteeism (0.39) large enough to not be considered as evidence to dismiss convergent validity.

\section{Known-groups validity}

Further evidence supporting the associations between WPAI domain scores and other health outcomes in UC patients was examined using the known-groups approach for discriminant validity. Differences in mean WPAI domain scores were calculated between subgroups of patients classified by health status in 11 comparisons from eight studies [30, 31, 34-37, 39, 41].

As reported in Table 5, across all studies, patients in subgroups defined by worse health outcomes (e.g., active disease; presence or lack of improvement in UC symptoms) scored clinically meaningfully worse on all WPAI domains than did patients in corresponding subgroups of better health outcomes. Summarized differences indicate that patients with worse health outcomes scored approximately $20-25 \%$ higher (worse) on presenteeism, OWI, and activity impairment domains, and approximately $12 \%$ worse on the absenteeism domain, than did patients with better health outcomes. Differences exceeded the CIC threshold of $7 \%$ for absenteeism in eight of the 11 comparisons, presenteeism in 10 of the 11 comparisons, and OWI and activity impairment in all comparisons.

Table 4 Convergent validity: Correlations between WPAI domains and criterion measures of disease activity

\begin{tabular}{|c|c|c|c|c|c|}
\hline \multirow[b]{2}{*}{ Study } & \multirow[b]{2}{*}{ Criterion Measure } & \multicolumn{4}{|l|}{ WPAI Domain } \\
\hline & & Absenteeism & Presenteeism & OWI & Activity Impairment \\
\hline Jackson 2016 [38] & SCCAI & - & 0.76 & 0.85 & 0.83 \\
\hline Mandel 2014 [32] & Partial Mayo score & 0.45 & 0.51 & 0.60 & 0.37 \\
\hline Yarlas 2014 [41] & UC-DAI & 0.32 & 0.34 & 0.42 & 0.43 \\
\hline Median correlation & & 0.39 & 0.51 & 0.60 & 0.43 \\
\hline
\end{tabular}

OW, Overall Work Impairment; SCCAI Simple Clinical Colitis Activity Index; UC-DAI Ulcerative Colitis - Disease Activity Index; WPAI Work Productivity and Activity Impairment questionnaire

-- Value not reported

Values printed in BOLD indicate correlations satisfying the definition of convergent validity $(\geq|0.40|)[24]$ 
Table 5 Differences in WPAI scores between UC patient subgroups differing on health status

\begin{tabular}{|c|c|c|c|c|c|c|c|}
\hline \multirow[b]{2}{*}{ Study } & \multirow[b]{2}{*}{$\mathrm{N}$} & \multirow[b]{2}{*}{ Predictor } & \multirow[b]{2}{*}{ Comparison } & \multicolumn{4}{|c|}{ Group mean differences in WPAI domain scores } \\
\hline & & & & Absenteeism & Presenteeism & OWI & $\begin{array}{l}\text { Activity } \\
\text { Impairment }\end{array}$ \\
\hline Gibson 2014 [31] & 175 & Remission status (partial Mayo) & Active vs Remission & 20.3 & 13.4 & 17.2 & 27.8 \\
\hline Vaizey 2014 [34] & 173 & Remission status (partial Mayo) & Active vs Remission & 22.8 & 21.2 & 26.4 & 29.7 \\
\hline $\begin{array}{l}\text { Katz Avitan } 2016 \\
\text { [39] }\end{array}$ & 150 & Remission status (SCCAI) & Active vs Remission & 24.5 & 36.7 & 42.1 & - \\
\hline $\begin{array}{l}\text { van Assche } 2015 \\
\text { [35] }\end{array}$ & 242 & Remission status (self-perceived) & Active vs Remission & 10.4 & 22.0 & 23.0 & 24.0 \\
\hline Yarlas 2015b [37] & 180 & Remission status, Month 12 (UC-DAl) & Active vs Remission & 11.7 & 25.6 & 34.3 & 26.5 \\
\hline Yarlas 2015b [37] & 258 & Remission status, Week 8 (UC-DAl) & Active vs Remission & 7.4 & 22.6 & 26.7 & 26.0 \\
\hline Yarlas 2014 [41] & 180 & Remission status (UC-DAl) & Active vs Remission & 5.0 & 5.3 & 11.1 & 9.7 \\
\hline Yarlas 2014 [41] & 180 & Improved rectal bleeding (UC-DAI) & $\begin{array}{l}\text { Not improved vs } \\
\text { improved }\end{array}$ & 6.3 & 7.8 & 12.2 & 12.3 \\
\hline Yarlas 2014 [41] & 180 & Improved stool frequency (UC-DAI) & $\begin{array}{l}\text { Not improved vs } \\
\text { improved }\end{array}$ & 12.5 & 13.8 & 20.0 & 19.2 \\
\hline Yarlas 2015a [36] & 146 & $\begin{array}{l}\text { Clinical symptom recurrence (UC- } \\
\text { DAI) }\end{array}$ & $\begin{array}{l}\text { Recurrent vs Non- } \\
\text { recurrent }\end{array}$ & -0.9 & 12.7 & 12.5 & 9.2 \\
\hline Cohen 2014 [30] & 95 & Fatigue (FACIT-Fatigue) & Fatigue vs Non-fatigue & 11.3 & 23.9 & 23.8 & 25.7 \\
\hline \multicolumn{4}{|c|}{ Weighted mean difference ${ }^{a}$} & 11.7 & 18.5 & 22.7 & 21.3 \\
\hline \multicolumn{4}{|l|}{ Median difference } & 11.3 & 21.2 & 23.0 & 24.9 \\
\hline \multicolumn{4}{|c|}{ Percentage of values exceeding $\mathrm{CIC}$} & $73 \%$ & $91 \%$ & $100 \%$ & $100 \%$ \\
\hline
\end{tabular}

CIC clinically important change; FACIT Functional Assessment of Chronic Illness Therapy; OWI Overall Work Impairment; SCCAI Simple Clinical Colitis Activity Index; UC, ulcerative colitis; UC-DAI Ulcerative Colitis - Disease Activity Index; WPAI Work Productivity and Activity Impairment questionnaire

aeighted by sample size

--Reported value were not within the valid range (i.e., was $>100$ )

Values printed in BOLD indicate mean differences exceeding the $\mathrm{CIC}$ threshold of $7 \%$ [27]

\section{Ability to detect change}

The ability of WPAI domains to detect changes in underlying UC disease activity was evaluated by assessing the magnitude of change in WPAI domain mean scores for patients showing clinically meaningful changes in activity status (i.e., a change from active disease to remission, or vice-versa). One study compared assessments of mean WPAI domain scores from an open-label treatment study for two groups of patients: 1) patients with active UC who achieved remission following eight weeks of once-daily treatment with multimatrix mesalamine $4.8 \mathrm{~g} /$ day and 2) patients with UC in remission who relapsed after 12 months in an extension phase during which they received once-daily treatment with multimatrix mesalamine $2.4 \mathrm{~g} /$ day once daily for 12 months [37].

As reported in the top row of Table 6, patients with active disease who achieved remission at Week 8 reported an approximately $25-30 \%$ decrease (i.e., improvement) in presenteeism, OWI, and activity impairment and an approximately 9\% decrease in absenteeism, all exceeding thresholds indicating clinically meaningful change. On the other hand, patients in the extension phase who relapsed at Month 12 reported an approximately $20-25 \%$ increase in presenteeism, OWI, and activity impairment, and an approximately 9\% increase in absenteeism, all exceeding thresholds indicating clinically meaningful change. These results indicate

Table 6 Changes in WPAl scores over time as a function of change in UC disease activity status

\begin{tabular}{|c|c|c|c|c|c|c|c|c|}
\hline \multirow[b]{2}{*}{ Study } & \multirow[b]{2}{*}{$\mathrm{N}$} & \multirow[b]{2}{*}{ Time 1 status } & \multirow[b]{2}{*}{ Time 2 status } & \multirow[b]{2}{*}{ Interval } & \multicolumn{4}{|c|}{ Change in mean WPAI scores from Time 1 to Time 2} \\
\hline & & & & & Absenteeism & Presenteeism & OWl & Activity Impairment \\
\hline Yarlas 2015b [37] & 180 & Active & Remission & 8 weeks $^{a}$ & -8.7 & -26.0 & -28.0 & -30.1 \\
\hline Yarlas 2015b [37] & 258 & Remission & Active & 12 months $^{\mathrm{b}}$ & 9.4 & 19.4 & 25.4 & 22.3 \\
\hline
\end{tabular}

OWI Overall Work Impairment; UC ulcerative colitis; WPAI Work Productivity and Activity Impairment questionnaire

${ }^{\text {a }}$ Receiving multimatrix mesalamine $4.8 \mathrm{~g} /$ day once daily

${ }^{\mathrm{b}}$ Receiving multimatrix mesalamine $2.4 \mathrm{~g} /$ day once daily

Remission defined as UC-DAl total score $\leq 1$, rectal bleeding and stool frequency components $=0$, and $\geq 1$-point reduction in endoscopy score

from induction phase baseline

Values printed in BOLD indicating those exceeding established thresholds indicating clinically important change 
the ability of WPAI domains to detect both positive change and negative change in patients' UC activity.

\section{Responsiveness to treatment}

The responsiveness of WPAI domains to effective treatment was evaluated by assessing the magnitude of change in WPAI domain mean scores reported at pre-treatment and post-treatment visits in studies using non-comparative treatment intervention designs. The change in WPAI domain scores over eight weeks from two prospective open-label studies of multimatrix mesalamine treatment (one administering $2.4-4.8 \mathrm{~g} /$ day once daily, [36] the other $4.8 \mathrm{~g} /$ day once daily [40]) and one prospective open-label study of adalimumab treatment (160/80 mg at Weeks $0 / 2$ followed by $40 \mathrm{mg}$ every other week through Week 26) [29] was evaluated. Results based on primary efficacy analyses of each study showed that treatment was effective in inducing clinical response and remission [29, 47].

As reported in Table 7, summaries of change following treatment across these three studies indicate that patients reported an approximately 20\% decrease in presenteeism, OWI, and activity impairment and an $8 \%$ decrease in absenteeism, all exceeding thresholds indicating clinically meaningful change.

\section{Discussion}

This first review (to our knowledge) of the measurement properties of the WPAI when used with UC patients shows the instrument to be reliable, valid, able to detect change, and responsive to treatment when used to assess WRO in this patient population. Results from reviewed studies found evidence that WPAI domain scores were reproducible after 12 months in patients who showed no change in underlying disease condition, and showed change in the expected direction among patients whose disease status improved (e.g., achieved remission) or worsened (e.g., relapsed) over time.
Two kinds of evidence supported the validity of the WPAI. First, convergent validity was supported by findings of higher scores on WPAI domains, particularly presenteeism, OWI, and activity impairment, being associated with lower scores on measures of HRQoL and with higher scores on indices of disease activity - that is, worse scores on WPAI domains were associated with worse scores on health status and disease activity, as expected. Second, known-groups validity was supported by findings that WPAI scores were substantially higher for patients with worse disease activity and more severe symptoms, again as expected. Divergent validity was not examined in this review, due to a lack of relevant data from identified sources.

Ability to detect change was supported by evidence of decreases in WPAI scores for patients with improved disease status (i.e., patients in active disease who achieved remission) and by increases in WPAI scores for patients with worsened disease status (i.e., patients in remission who relapsed to active disease). Responsiveness to treatment was evidenced by substantial decreases in scores for patients who received effective treatments. The magnitude of differences in scores over time and by condition exceeded established CIC thresholds, indicating that changes and differences were clinically meaningful.

Measurement properties were weaker for the WPAI absenteeism domain relative to the other three domains. This is likely related to the highly skewed distribution observed for the absenteeism domain in most studies, with the majority of responses indicating zero days absent in the past seven days due to health problems. For example, in Yarlas et al. (2015a) [36], a response of zero was observed in $73 \%$ of subjects with active UC (as compared to approximately $25 \%$ in the other three domains), meaning that positive change (reduction) in the absenteeism domain was only possible for one-quarter of subjects. This limits the magnitude of improvements that can be observed as a function of

Table 7 Responsiveness to treatment: change in WPAI scores following effective treatment interventions in prospective, open-label studies

\begin{tabular}{|c|c|c|c|c|c|c|c|}
\hline \multirow[b]{2}{*}{ Study } & \multirow[b]{2}{*}{$\mathrm{N}$} & \multirow[b]{2}{*}{ Treatment regimen } & \multirow[b]{2}{*}{ Treatment duration } & \multicolumn{4}{|c|}{ Change (mean WPAI scores at Week 8 - mean WPAI scores at baseline) } \\
\hline & & & & Absenteeism & Presenteeism & OWI & Activity Impairment \\
\hline Travis 2017 [29] & 446 & $\begin{array}{l}\text { ADA (initial } 160 / 80 \mathrm{mg} \text {, } \\
40 \mathrm{mg} \text { EOW at Week 4) }\end{array}$ & 26 weeks & -11.4 & -24.5 & -29.2 & -27.2 \\
\hline Willshire 2014 [40] & 404 & $\begin{array}{l}\text { MMX mesalamine } \\
4.8 \mathrm{~g} / \text { day }\end{array}$ & 8 weeks & -7.6 & -20.6 & -23.4 & -24.1 \\
\hline Yarlas 2015a [36] & 103 & $\begin{array}{l}\text { MMX mesalamine } \\
2.4-4.8 \mathrm{~g} / \text { day }\end{array}$ & 8 weeks & -4.0 & -12.3 & -13.8 & -14.2 \\
\hline \multicolumn{3}{|c|}{ Weighted mean change ${ }^{a}$} & & -9.0 & -21.5 & -25.1 & -24.5 \\
\hline \multicolumn{3}{|l|}{ Median change } & & -7.6 & -20.6 & -23.4 & -24.1 \\
\hline
\end{tabular}

ADA adalimumab, EOW every other week, MMX multimatrix, OWI Overall Work Impairment, WPAI Work Productivity and Activity Impairment questionnaire Values printed in BOLD indicating those exceeding established thresholds indicating clinically important change

aeighted by sample size 
treatment or accompanying change in disease status, and the restricted range also can lead to underestimation of correlations with other variables [48].

The magnitudes of association for WPAI domains with criterion HRQoL measures varied across the domains of those measures. Specifically, all WPAI domains showed stronger associations with the SIBDQ Bowel symptoms and Social function domains than with the Systemic symptoms and Emotional function domains. Most WPAI domains also correlated more strongly with the SF-12v2 Bodily pain, Role physical, and Role emotional domains than other domains of the SF-12v2. These patterns of associations are logical from both a clinical and content perspective. From a clinical perspective, bowel symptoms, such as stool urgency and abdominal pain, would lead to impairment in work and activity impairment more than mental health or perception of general health. From a content standpoint, the SIBDQ Social function domain and the SF-12v2 Role physical and Role emotional domains assess constructs involving the impact of health on work and other activities, and thus would be expected to strongly correlate with WPAI domains. So, while divergent validity was not explicitly examined in this review, the patterns of magnitudes of convergence with HRQoL domains are consistent with content overlap.

The majority of studies included in this review reported administering the UC-specific version of the WPAI (i.e., WPAI-UC), with only three studies [29, 30, 38] reporting use of the general health version (i.e., WPAI-GH). One would expect more precision in findings from studies using the WPAI-UC than the WPAI-GH, since the former is more specific to UC activity, but in fact this was not observed in our review, as findings were comparable across both types of instruments. For studies using the WPAI-GH, this may be indicative of the fact that UC accounted for the majority of health problems in these patients, or that within the context of these studies, where the focus was on patients' UC, patients responses were driven by their perceptions of their UC-specific health even without explicit instruction. However, to maximize the sensitivity of the instrument, we recommend that the disease-specific version be used as a trial endpoint.

There are some limitations in the current review and gaps in the extant literature that require discussion. First, the evidence base was rather small. Evidence for test-retest reliability, ability to detect change, and convergence with HRQoL measures were each based on findings from a single study, while evidence for convergence with measures of disease activity and responsiveness to treatment were based on only three studies each.

The evidence base was limited due the lack of potentially relevant data. We conducted a systematic, comprehensive search of both the published and unpublished literature. However, our search of the unpublished 'gray' literature was focused on presentations and posters at conferences deemed relevant to the topic and accessible through the Embase database (as well as the ISPOR conference). Further, as discussed earlier, there were two dozen conference presentations that we were unable to retrieve, either due to the authors' refusal to provide or our inability to get a response from authors even after repeated contact attempts. Assuming that the availability of presentations was unrelated to the findings reported (which was supported by the titles and abstracts of unavailable presentations), then the findings presented here should be unbiased and generalizable.

Another point for consideration is the quality of the studies included in this review. While all 13 identified studies were judged as acceptable for inclusion during our quality assessment process, most of these studies, or at least their description of them, had weaknesses identified during this process. Most of the posters reported only cursory descriptions of the study sample, limiting the ability to generalize their findings to a larger patient population. None of the studies provided any justification for sample size; however, since we do not consider statistical significance of findings in our review, but rather effect sizes or magnitudes of differences/change, this limitation is not too relevant to our findings.

All but one of the 13 studies failed to make statistical adjustments or use another strategy to identify or control for confounding factors in their statistical models, which was an item on both the JBI and NIH checklists. This failure to control for possible confounding factors means that the effect sizes and magnitude of differences and change in our assessments of validity, ability to detect change, and responsiveness may be biased. Findings from this review of evidence in the literature is bound by the quality of that evidence. While no study was judged to be of poor enough quality that required exclusion from the review, all reviewed studies were flawed in some way, and so the possibility of bias or limits to the generalizability of our findings cannot be fully dismissed.

We examined the responsiveness of the WPAI to effective treatment in observational studies, but did not identify publications of randomized-controlled trials (RCTs) with UC patients in which the WPAI was included as an endpoint. This limited our ability to examine its responsiveness (within-patient change) and sensitivity to detect treatment differences (between-group difference) in RCTs. We are aware of recent, unpublished RCTs with UC patients in which the WPAI was administered, and hope that results from these studies will be made available soon so that the instrument can be evaluated for this purpose.

Another important gap in the existing literature is that no identified studies have solely examined the 
measurement properties of the WPAI. The review here relied on use of the WPAI data in studies that had other research purposes. A study dedicated to examining the psychometric profile of the WPAI in a large UC sample would provide a much needed base of information regarding these properties.

\section{Conclusion}

This review found that the WPAI has demonstrated good measurement properties in studies of UC patients. Findings from these studies included evidence that WPAI domains have adequate test-retest reliability; convergent validity with measures of HRQoL and UC disease activity; discriminant validity in predicting classification of patients by UC disease activity or other health-related outcomes; ability to detect changes in disease activity; and evidence of responsiveness to effective treatment. At the same time, because of the limited evidence base in the published and unpublished literature, these findings should be considered encouraging though preliminary until more evidence emerges.

\section{Additional file}

Additional file 1: Appendix 1. Work Productivity and Activity Impairment Questionnaire (WPAI). Appendix 2. Terms and Strings Used in Electronic Database Literature Searches. (DOCX 21 kb)

\section{Abbreviations \\ CDAl: Crohn's Disease Activity Index; CENTRAL: Cochrane Register of Controlled Trials; CIC: Clinically Important Change; HRQoL: Health-related Quality of Life; IBD: Inflammatory Bowel Disease; ISPOR: International Society for Pharmacoeconomics and Outcomes Research; OWl: Overall Work Impairment; PRISMA: Preferred Reporting Items for Systematic Reviews and Meta-Analyses; RCT: Randomized Controlled Trial; SCCAl: Simple Clinical Colitis Activity Index; SF-12v2: SF-12v2 ${ }^{\circledR}$ Health Survey; SIBDQ: Short Inflammatory Bowel Disease Questionnaire; UC: Ulcerative Colitis; UC- DAI: Ulcerative Colitis Disease Activity Index; WPAI: Work Productivity and Activity Impairment; WPAl:UC: Work Productivity and Activity Impairment, Ulcerative Colitis-Specific Version; WPAI-CD: Work Productivity and Activity Impairment, Crohn's Disease-Specific Version; WPAI-GH: Work Productivity and Activity Impairment, General Health Version; WRO: Work-Related Outcomes}

\section{Acknowledgements}

Not applicable.

\section{Availability of data and supporting materials}

Data sharing not applicable to this article as no datasets were generated or analysed during the current study.

\section{Funding}

This literature review and synthesis and the writing of this manuscript was funded by Pfizer Inc.

\section{Authors' contributions}

AY conceptualized the study, contributed to the literature review and synthesis, and drafted the manuscript. SM contributed to the literature review and synthesis and provided critical scientific input to the manuscript. MB conceptualized the study and provided critical scientific input to the manuscript. AL contributed to the literature review and synthesis and provided critical scientific input to the manuscript. JC conceptualized the study and provided critical scientific input to the manuscript. MD provided critical scientific input to the manuscript. All authors read and approved the final manuscript.

Ethics approval and consent to participate

Not applicable.

Consent for publication

Not applicable.

\section{Competing interests}

$\mathrm{JC}$ and MD are employees and shareholders of Pfizer Inc., which funded the study and manuscript. AY, SM, MB, and AL are employees of Optum, which received consulting fees from Pfizer to conduct this research and develop this manuscript

\section{Publisher's Note}

Springer Nature remains neutral with regard to jurisdictional claims in published maps and institutional affiliations.

\section{Author details}

1Optum, 1301 Atwood Avenue, Suite 311N, Johnston, RI 02919, USA. ²Pfizer, 445 Eastern Point Road, MS 8260-2502, Groton, CT 06340, USA. ${ }^{3}$ Pfizer, 235 42nd Street, New York, NY 10017, USA.

Received: 23 July 2018 Accepted: 30 November 2018 Published online: 13 December 2018

\section{References}

1. Cosnes, J., Gower-Rousseau, C., Seksik, P., et al. (2011). Epidemiology and natural history of inflammatory bowel diseases. Gastroenterology, 140, 1785-1794.

2. Danese, S., \& Fiocchi, C. (2011). Ulcerative colitis. The New England Journal of Medicine, 365, 1713-1725.

3. Schroeder, K. W., Tremaine, W. J., \& Ilstrup, D. M. (1987). Coated oral 5aminosalicylic acid therapy for mildly to moderately active ulcerative colitis. A randomized study. The New England Journal of Medicine, 317, 1625-1629.

4. Walmsley, R. S., Ayres, R. C., Pounder, R. E., et al. (1998). A simple clinical colitis activity index. Gut, 43, 29-32.

5. Devlen, J., Beusterien, K., Yen, L., et al. (2014). The burden of inflammatory bowel disease: A patient-reported qualitative analysis and development of a conceptual model. Inflammatory Bowel Diseases, 20, 545-552.

6. Jansen, F., van Uden-Kraan, C. F., Braakman, J. A., et al. (2015). A mixedmethod study on the generic and ostomy-specific quality of life of cancer and non-cancer ostomy patients. Support Care Cancer, 23, 1689-1697.

7. McCormick, J. B., Hammer, R. R., Farrell, R. M., et al. (2012). Experiences of patients with chronic gastrointestinal conditions: In their own words. Health and Quality Life Outcomes, 10, 25.

8. Sammut, J., Scerri, J., \& Xuereb, R. B. (2015). The lived experience of adults with ulcerative colitis. Journal of Clinical Nursing, 24, 2659-2667.

9. Waljee, A. K., Joyce, J. C., Wren, P. A., et al. (2009). Patient reported symptoms during an ulcerative colitis flare: A qualitative focus group study. European Journal of Gastroenterology \& Hepatology, 21, 558-564.

10. Wolfe, B. J., \& Sirois, F. M. (2008). Beyond standard quality of life measures: The subjective experiences of living with inflammatory bowel disease. Quality of Life Research, 17, 877-886.

11. Bernklev, T., Jahnsen, J., Henriksen, M., et al. (2006). Relationship between sick leave, unemployment, disability, and health-related quality of life in patients with inflammatory bowel disease. Inflammatory Bowel Diseases, 12 , 402-412.

12. Bernstein, C. N., Kraut, A., Blanchard, J. F., et al. (2001). The relationship between inflammatory bowel disease and socioeconomic variables. The American Journal of Gastroenterology, 96, 2117-2125.

13. Boonen, A., Dagnelie, P. C., Feleus, A., et al. (2002). The impact of inflammatory bowel disease on labor force participation: Results of a population sampled case-control study. Inflammatory Bowel Diseases, 8, 382-389.

14. Gibson, T. B., Ng, E., Ozminkowski, R. J., et al. (2008). The direct and indirect cost burden of Crohn's disease and ulcerative colitis. Journal of Occupational and Environmental Medicine, 50, 1261-1272. 
15. Longobardi, T., Jacobs, P., Wu, L., et al. (2003). Work losses related to inflammatory bowel disease in Canada: Results from a National Population Health Survey. The American Journal of Gastroenterology, 98, 844-849.

16. Reinisch, W., Sandborn, W. J., Bala, M., et al. (2007). Response and remission are associated with improved quality of life, employment and disability status, hours worked, and productivity of patients with ulcerative colitis. Inflammatory Bowel Diseases, 13, 1135-1140.

17. Reilly MC, Zbrozek AS, Dukes EM (1993) The validity and reproducibility of a work productivity and activity impairment instrument. PharmacoEconomics 4:353-365.

18. Bushnell, D. M., Reilly, M. C., Galani, C., et al. (2006). Validation of electronic data capture of the irritable bowel syndrome--quality of life measure, the work productivity and activity impairment questionnaire for irritable bowel syndrome and the EuroQol. Value Health, 9, 98-105.

19. Reilly, M. C., Bracco, A., Ricci, J., et al. (2004). The validity and accuracy of the work productivity and activity impairment questionnaire--irritable bowel syndrome version (WPAI:IBS). Alimentary Pharmacology \& Therapeutics, 20, 459-467.

20. Wahlqvist, P., Carlsson, J., Stålhammar, N., et al. (2002). Validity of a work productivity and activity impairment questionnaire for patients with symptoms of gastro-esophageal reflux disease (WPAI-GERD)--results from a cross-sectional study. Value Health, 5, 106-113.

21. Reilly, M. C., Gerlier, L., Brabant, Y., et al. (2008). Validity, reliability, and responsiveness of the work productivity and activity impairment questionnaire in Crohn's disease. Clinical Therapeutics, 30, 393-404

22. Vergara, M., Montserrat, A., Casellas, F., et al. (2011). A new validation of the Spanish work productivity and activity impairment questionnaire-Crohn's disease version. Value Health, 14, 859-861.

23. Moher, D., Liberati, A., Tetzlaff, J., et al. (2009). Preferred reporting items for systematic reviews and meta-analyses: The PRISMA statement. Open medicine, 3, e123-e130.

24. Cappelleri JC, Zou KH, Bushmakin AG et al. (2013) Patient-reported outcomes: Measurement, implementation and interpretation [chapter 3: Validity]. Chapman \& Hall/CRC Press, Boca Raton, FL.

25. Jaeschke, R., Singer, J., \& Guyatt, G. H. (1989). Measurement of health status. Ascertaining the minimal clinically important difference. Controlled Clinical Trials, 10, 407-415

26. Best, W. R., Becktel, J. M., Singleton, J. W., et al. (1976). Development of a Crohn's disease activity index. National Cooperative Crohn's Disease Study. Gastroenterology, 70, 439-444.

27. Reilly MC, Brown M, Brabant $Y$ et al. (2007) Minimally important difference for WPAl:CD scores: Defining relevant impact on work productivity in active Crohn's disease (Abst 962). American College of Gastroenterology Annual Scientific Meeting, Philadelphia.

28. Binion, D. G., Louis, E., Oldenburg, B., et al. (2011). Effect of adalimumab on work productivity and indirect costs in moderate to severe Crohn's disease: A meta-analysis. Canadian Journal of Gastroenterology, 25, 492-496.

29. Travis, S., Feagan, B. G., Peyrin-Biroulet, L., et al. (2017). Effect of adalimumab on clinical outcomes and health-related quality of life among patients with ulcerative colitis in a clinical practice setting: Results from InspirADA. Journal of Crohn's \& Colitis, 11, 1317-1325.

30. Cohen, B. L., Zoega, H., Shah, S. A., et al. (2014). Fatigue is highly associated with poor health-related quality of life, disability and depression in newlydiagnosed patients with inflammatory bowel disease, independent of disease activity. Alimentary Pharmacology \& Therapeutics, 39, 811-822.

31. Gibson, P. R., Vaizey, C., Black, C. M., et al. (2014). Relationship between disease severity and quality of life and assessment of health care utilization and cost for ulcerative colitis in Australia: A cross-sectional, observational study. Journal of Crohn's \& Colitis, 8, 598-606.

32. Mandel, M. D., Balint, A., Lovasz, B. D., et al. (2014). Work disability and productivity loss in patients with inflammatory bowel diseases in Hungary in the era of biologics. The European Journal of Health Economics, 15(Suppl 1), S121-S128.

33. Meijs S, Gardenbroek TJ, Sprangers M et al. (2014) Health-related quality of life and disability in patients with ulcerative colitis and proctocolectomy with ileoanal pouch versus treatment with anti-TNF agents. Journal Crohn's \& Colitis 8:686-692.

34. Vaizey, C. J., Gibson, P. R., Black, C. M., et al. (2014). Disease status, patient quality of life and healthcare resource use for ulcerative colitis in the UK: An observational study. Frontline Gastroenterology, 5, 183-189.
35. van Assche, G., Peyrin-Biroulet, L., Sturm, A., et al. (2016). Burden of disease and patient-reported outcomes in patients with moderate to severe ulcerative colitis in the last 12 months - multicenter European cohort study. Digestive and Liver Disease, 48, 592-600.

36. Yarlas, A., Yen, L., \& Hodgkins, P. (2015). The relationship among multiple patient-reported outcomes measures for patients with ulcerative colitis receiving treatment with $M M X$ formulated delayed-release mesalamine. Quality of Life Research, 24,671-683.

37. Yarlas A, D'Haens G, Willshire D et al. (2015) Remission status predicts workrelated outcomes for patients with mild-to-moderate ulcerative colitis receiving short-term and long-term daily therapy with multimatrix mesalamine. American journal of gastroenterology conference 80th annual scientific meeting of the American College of Gastroenterology. Honolulu HI United States. Conference start 20151016. Conference end 20151021. Conference publication:S821.

38. Jackson B, Con D, Gorelik A et al. (2016) Examination of the relationship between disease activity, psychological distress and patientreported outcome measures in an inflammatory bowel disease cohort. Journal of Crohn's and colitis conference 11th congress of the European Crohn's and colitis organisation, ECCO 2016. Netherlands. Conference start 20160316. Conference end 20160319. 10:S467.

39. Katz Avitan, A., Schwartz, D., Greenberg, D., et al. (2016). The impact of inflammatory bowel disease on work productivity: Comparison of a realworld sample of patients with Crohn's disease and ulcerative colitis. Value Health, 19, A511.

40. Willshire, D., Willian, M. K., Yarlas, A., et al. (2014). The effectiveness of shortterm and long-term daily treatment with multimatrix mesalazine on improving work-related outcomes in adult patients with mild-to-moderate ulcerative colitis. Journal of Gastroenterology and Hepatology, 29, 107.

41. Yarlas, A., Willian, M., \& Joshi, A. V. (2014). The correspondence between patient-reported outcome (PRO) instruments and measures of disease activity in adult patients with mild-to-moderate ulcerative colitis (UC) receiving short-term daily treatment with $\mathrm{mmx}$ mesalamine. Value Health, 17, A40.

42. Joanna Briggs Institute: Critical Appraisal Tools: Checklist for Analytical Cross Sectional Studies. Joanna Briggs Institute. Available online at http:// joannabriggs.org/research/critical-appraisal-tools.html. Accessed 17 Dec 2017.

43. National Institutes of Health Quality Assessment Tool for Observational Cohort and Cross-Sectional Studies. https://www.nhlbi.nih.gov/healthtopics/study-quality-assessment-tools. Accessed 17 Dec 2017.

44. Sutherland, L. R., Martin, F., Greer, S., et al. (1987). 5-Aminosalicylic acid enema in the treatment of distal ulcerative colitis, proctosigmoiditis, and proctitis. Gastroenterology, 92, 1894-1898.

45. Irvine, E. J., Zhou, Q., \& Thompson, A. K. (1996). The short inflammatory bowel disease questionnaire: A quality of life instrument for community physicians managing inflammatory bowel disease. CCRPT investigators. Canadian Crohn's relapse prevention trial. The American Journal of Gastroenterology, 91, 1571-1578.

46. Maruish ME (ed) (2012) User's manual for the SF-12v2 survey (3rd edition). QualityMetric, Incorporated, Lincoln, Rl.

47. Rubin, D. T., Bradette, M., Gabalec, L., et al. (2016). Ulcerative colitis remission status after induction with Mesalazine predicts maintenance outcomes: The MOMENTUM trial. Journal of Crohn's \& Colitis, 10, 925-933.

48. Sackett, P. R., \& Yang, H. (2000). Correction for range restriction: An expanded typology. Journal of Applied Psychology, 85, 112-118. 